\title{
RINGS ALL OF WHOSE TORSION QUASI-INJECTIVE MODULES ARE INJECTIVE
}

\author{
by J. AHSAN and E. ENOCHS
}

(Received 26 April, 1983)

1. Introduction and background. Throughout this paper it is assumed that rings are associative, have the identity element, and all modules are left unital. $R$ will denote a ring with identity, $R$-Mod the category of left $R$-modules, and for each left $R$-module $M$, $E(M)$ (resp. $J(M)$ ) will represent the injective hull (resp. Jacobson radical) of $M$. Also, for a module $M, A \subseteq^{\prime} M$ will mean that $A$ is an essential submodule of $M$, and $Z(M)$ denotes the singular submodule of $M . M$ is called singular if $Z(M)=M$, and it is called non-singular in case $Z(M)=0$. For fundamental definitions and results related to torsion theories, we refer to [12] and [14]. In this paper we shall deal mainly with Goldie torsion theory. Recall that a pair $(G, F)$ of classes of left $R$-modules is known as Goldie torsion theory if $G$ is the smallest torsion class containing all modules $B / A$, where $A \subseteq B$, and the torsion free class $F$ is precisely the class of non-singular modules.

A ring $R$ is called a left $V$-ring if each simple left $R$-module is injective. $R$ is a left $V$-ring if and only if each left $R$-module has zero Jacobson radical (if and only if each left ideal of $R$ is the intersection of maximal left ideals) (see [13, Theorem 2.1]). In the commutative case, it is well known that $R$ is a V-ring if and only if $R$ is regular (in the sense of von Neumann). A module $M$ is quasi-injective if every homomorphism from a submodule of $M$ into $M$ can be lifted to an endomorphism of $M$. A ring $R$ is called a left $Q I$-ring if each quasi-injective left $R$-module is injective. These rings were originally introduced in [1], and later studied by many authors (see, for example, $[2,3,4,6,7,8$, 10]). Left QI-rings are left Noetherian and left V-rings (see [1]). Thus commutative QI-rings are semisimple Artinian. The goal of this paper is to study rings all of whose (Goldie) torsion quasi-injective modules are injective. The results developed in the next section indicate that these rings provide an effective torsion theoretical generalization of left QI-rings.

2. Results. Let $(T, F)$ be a hereditary torsion theory, and let $\mathscr{F}(T)$ be the associated filter of left ideals of $R$. A left ideal of $R$ which is a member of $\mathscr{F}(T)$ will be called an $\mathscr{F}$-ideal. Relative to $(T, F)$, a left $R$-module $E$ is called $T$-injective if $\operatorname{Ext}_{R}(R / I, E)=0$ for all $I \in \mathscr{F}(T)$. This definition is equivalent to the following statement: For any $I \in \mathscr{F}(T)$, each homomorphism $f: I \rightarrow E$ can be extended to a homomorphism $g: R \rightarrow E$. The following result concerning $T$-injective modules can be found in Golan and Teply [11, Lemma 2, p. 252].

LEMMA 1. Let $(T, F)$ be a hereditary torsion theory for $R$-Mod. Then the following are equivalent:

(1) $R$ has ACC on F्F-ideals;

(2) any direct sum of (countably many) T-injective torsion modules is T-injective.

Glasgow Math. J. 25 (1984) 219-227. 
We now consider the Goldie torsion theory $(G, F)$ for $R$-Mod, whose associated filter of left ideals is denoted by $\mathscr{F}(G)$. Since all essential left ideals of $R$ belong to $\mathscr{F}(G)$, it follows that, relative to the Goldie torsion theory, a left $R$-module is $T$-injective if and only if it is injective. Thus the following proposition is immediate in view of the above lemma.

Propostrion 1. Let $(G, F)$ be the Goldie torsion theory for $R$-Mod. Then $R$ has ACC on F-ideals if and only if each direct sum of torsion injective modules is injective.

We now phrase a definition for the sake of brevity.

DefinITION. A ring $R$ will be called a left TQI-ring if each torsion quasi-injective left $R$-module is injective.

Thus every left QI-ring is left TQI. The converse, however, is not true in general (see Example 1 below). We now prove some lemmas concerning left TQI-rings. Throughout the remainder of this paper, it will be assumed that we are working in the context of Goldie torsion theory.

Lemma 2. Let $R$ be a left TQI-ring. Then for each torsion left $R$-module $M, J(M)=0$.

Proof. Let $M$ be a torsion left $R$-module. Let $x \in M, x \neq 0$. Then, by Zorn's lemma, there is a submodule $Y$ of $M$ which is maximal among the submodules $X$ of $M$ with $x \notin X$. Let $D=R x+Y$. Then $x \in D$, and $D / Y \neq(0)$. Also, $D / Y$ is simple and torsion. Hence $D / Y$ is injective. Therefore, $M / Y=D / Y \oplus K / Y$, where $K$ is a submodule of $M$. Since $x \notin K$, $M / Y=D / Y$. Hence $Y$ is a maximal submodule of $M$. Since $x \notin Y, J(M)=(0)$.

COROllary 1. Let $I$ be an $\mathscr{F}$-ideal of $R$. Then $I$ is an intersection of maximal left ideals.

Proof. Since $I$ is an $\mathscr{F}$-ideal, $R / I$ is a torsion left $R$-module. Therefore $J(R / I)=(0)$. Hence $I$ is an intersection of maximal left ideals.

COROLlaRy 2. If each $\mathscr{F}$-ideal is the intersection of maximal left ideals then each simple torsion left $R$-module is injective.

Proof. Let $S$ be a simple torsion left $R$-module, and let $I$ be an $F$-ideal. Suppose $f \in \operatorname{Hom}_{R}(I, S)$. We claim that $f$ is extendable to an element of $\operatorname{Hom}_{R}(R, S)$. Let $\operatorname{Ker} f=K$. Consider the sequence: $0 \rightarrow I / K \rightarrow R / K \rightarrow R / I \rightarrow 0$. Since $I / K$ and $R / I$ are torsion, $R / K$ is torsion. Thus both $K$ and $I$ are $\mathscr{F}$-ideals and $I \supseteq K$. If $I=K$ then we are done, otherwise there is a maximal left ideal $L$ of $R$ such that $L$ contains $K$, but does not contain $I$. Thus $L+I=R$ and $L \cap I=K$. Hence $R / K=L / K \oplus I / K$. Thus the above sequence splits and it can be shown that $f$ is extendable to a map from $R$ to $S$. Hence $S$ is $T$-injective, and so it is injective, as we are working in the Goldie torsion theory.

The proofs of Lemma 2, Corollaries 1 and 2 are adaptions from [13, Theorem 2.1].

Lemma 3. Let $R$ be a left TQI-ring. Then $R$ has ACC on F्F-ideals.

Proof. Let $I_{1} \subset I_{2} \subset \ldots \subset I_{n} \subset \ldots$ be an ascending chain of distinct $\mathscr{F}$-ideals of $R$. 
Then by Corollary 1 to Lemma 2 , there are maximal left ideals $M_{k}, k=1,2, \ldots$, such that $I_{k} \subset M_{k}$ but $I_{k+1} \not \subset M_{k}$. Let $\pi_{k}: R \rightarrow R / M_{k}$ be the natural projection. Also, let $I=\bigcup_{k=1}^{\infty} I_{k}$, and define $f: I \rightarrow \sum_{k=1}^{\infty} \oplus R / M_{k}$ by $f(x)=\sum_{k=1}^{\infty} \pi_{k}(x)$. Note that $x$ is an element of only a finite number of the $M_{k}$, for all $x \in I$, and $f$ is an epimorphism. Since $\sum_{k=1}^{\infty} \oplus R / M_{k}$ is semisimple and torsion, it is torsion quasi-injective and hence injective, as $R$ is a left TQI-ring. Hence $f$ extends to $g: R \rightarrow \sum_{k=1}^{\infty} \oplus R / M_{k}$. Since $R$ has an identity, $g(R)$ and hence $g(I)$ is contained in $\sum_{k=1}^{n} \oplus R / M_{k}$, for some positive integer $n$. This implies that the above chain of left $\mathscr{F}$-ideals is finite.

We now prove the following.

Theorem 1. Let $(G, F)$ be the Goldie torsion theory for $R$-Mod. Then the following are equivalent:

(1) $R$ is left TQI;

(2) each direct sum of torsion quasi-injective modules is quasi-injective.

Proof. Suppose $R$ is a left TQI-ring. Let $M=\bigoplus_{i} M_{i}$ be a direct sum of torsion quasi-injective modules $M_{i}$. Then each $M_{i}$ is injective by the hypothesis. Hence $M$ is injective by Proposition 1. Now, assume (2). Let $M$ be a torsion quasi-injective module. Then $M \oplus E(M)$ is quasi-injective, by the assumption. From this it follows that $M \cong E(M)$ and so $M$ is injective.

According to a well-known result, every injective module over a left Noetherian ring is the direct sum of indecomposable injective modules. A similar decomposition property is also true for quasi-injective modules over left Noetherian rings. The next two lemmas proved below provide analogues of these results for torsion injective and torsion quasiinjective modules over rings whose $\mathscr{F}$-ideals satisfy the ascending chain condition.

LEMMA 4. Let $R$ be a ring whose $\mathscr{F}$-ideals have ACC. Then every torsion injective left $R$-module is the direct sum of indecomposable injective modules.

Proof. Let $M$ be a torsion injective left $R$-module. Let $x \in M, x \neq 0$. Then $R x \cong R / I$, for some left ideal $I$ of $R$. Since $R x$ is torsion, $I$ is an $\mathscr{F}$-ideal. Furthermore, since $R$ has ACC on $\mathscr{F}$-ideals, it is easy to see that $R x$ is a Noetherian left $R$-module. Hence $R x$ contains a uniform submodule $U$. Since $E(U) \subseteq M, M$ has a torsion injective submodule which is the injective hull of its every non-zero submodule. Let $\left\{M_{i}\right\}_{j \in J}$ be a maximal independent family of submodules of $M$ such that each $M_{j}$ is the injective hull of all of its non-zero submodules, where $J$ is an indexing set. Now let $y \in M$ be a non-zero element. Then, as above, $E(R y)$ contains a submodule which is the injective hull of all of its non-zero submodules, and hence $\bigoplus_{j \in J} M_{j} \cap R y \neq(0)$. This implies that $\bigoplus_{j \in J} M_{j} \subseteq^{\prime} M$. On the other hand, since each $M_{j}$ is torsion injective and also since $R$ has ACC on $\mathscr{F}$-ideals, it 
follows from Proposition 1 that $\oplus M_{i}$ is injective. Hence $\oplus M_{j}$ is a direct summand of $M$. But $\underset{j \in J}{\bigoplus} M_{i} \subseteq^{\prime} M$. Therefore, $\underset{j \in J}{\bigoplus_{i \in J}} M_{j}=M$. Further, since the endomorphism ring of each indecomposable injective module is local, it follows from the Krull-Remak-Schmidt theorem that this decomposition is unique (up to isomorphism).

Lemma 5. Let $R$ be a ring whose $\mathscr{F}$-ideals have ACC. Then each torsion quasiinjective left $R$-module is the direct sum of indecomposable quasi-injective modules.

Proof. Let $M$ be a torsion quasi-injective left $R$-module. Then $E(M)$ is torsion, as we are dealing with Goldie torsion theory. Hence, by Lemma $4, E(M)=\underset{i}{\bigoplus_{i}} E_{i}$, where each $E_{j}$ is an indecomposable injective module. Let $p_{j}: E(M) \rightarrow E_{j}$ be the projection map. Then since $M$ is quasi-injective, $p_{j}(M) \subset M$. Now, let $x \in M$, say $x=\sum_{j} x_{j}, x_{j} \in E_{i}$; then $x_{j} \in M$. Then $M=\sum M \cap E_{j}$, i.e. $M=\sum \oplus\left(M \cap E_{j}\right)$. Hence $M$ is the direct sum of indecomposable quasi-injective modules.

Before we state the next theorem, we note that we shall use the notation $S(M)$ for the socle of a left $R$-module $M$. In particular, $S(R)$ will denote the socle of ${ }_{R} R$.

THEOREM 2. Let $R$ be a ring with $S(R) \subseteq^{\prime} R$, and $(G, F)$ be the Goldie torsion theory for $R$-Mod. Then the following are equivalent:

(1) $R$ is a left TQI-ring;

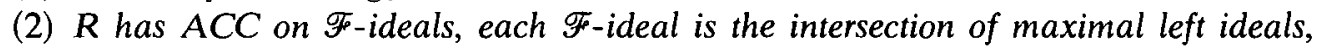
and $R / S(R)$ is a left QI-ring.

Proof. (1) Let us assume that $R$ is a left TQI-ring. Then, by Lemma $3, R$ has ACC

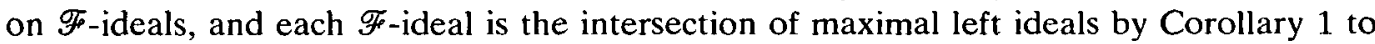
Lemma 2. Furthermore, since $S(R) \subseteq^{\prime} R$ by the hypothesis, $S(R)$ is an $\mathscr{F}_{\text {-ideal. Hence }}$ $R / S(R)$ is semiprime by Lemma 2 . Also, it follows immediately from Lemma 3 , that $R / S(R)$ is Noetherian. Moreover, since $R$ is a left TQI-ring, it is straightforward to argue that $R / S(R)$ is also a left TQI-ring. Now it is a known result that every semiprime left Noetherian left TQI-ring is a left QI-ring (see [2, Corollary 9, p. 48]). Hence $R / S(R)$ is a left QI-ring.

(2) Conversely, we now assume that $R$ has ACC on $\mathscr{F}$-ideals, each $\mathscr{F}$-ideal is the intersection of maximal left ideals, and $R / S(R)$ is a left QI-ring. We prove that $R$ is a left TQI-ring. So, let $M$ be a torsion quasi-injective left $R$-module. Then, by Lemma 5 , $M=\bigoplus_{\alpha \in \Lambda} M_{\alpha}$, where each $M_{\alpha}$ is torsion, indecomposable and quasi-injective. Consider an arbitrary but fixed $M_{\alpha}$. If $S\left(M_{\alpha}\right) \neq 0$ then there exists a simple (torsion) submodule $S(\neq 0)$ of $M$. Since each $\mathscr{F}_{\text {-ideal }}$ is the intersection of maximal left ideals, it follows from Corollary 2 to Lemma 2 that $S$ is injective. So, $S$ is a direct summand of $M_{\alpha}$. But $M_{\alpha}$ is an indecomposable module. Hence $M_{\alpha}=S$, i.e. $M_{\alpha}$ is injective. Let us now suppose that $S\left(M_{\alpha}\right)=0$. Since $M_{\alpha}$ is torsion, $M_{\alpha}$ may be regarded as an $R / S(R)$-module. Thus $M_{\alpha}$ is a quasi-injective $R / S(R)$-module. Since $R / S(R)$ is a left QI-ring, $M_{\alpha}$ is $R / S(R)$-injective. We claim that $M_{\mathrm{\alpha}}$ is $R$-injective. Let $I$ be a left ideal of $R$, and let $\alpha: I \rightarrow M_{\mathrm{\alpha}}$ be an $(R-)$ 
homomorphism. Let us define a map $\beta:(I+S(R)) / S(R) \rightarrow M_{\alpha}$, by the rule $\beta(x+S(R))=$ $\alpha(x)$, for all $x \in I$. If $x \in I \cap S(R)$ then $\alpha(x) \in \alpha(S(I)) \subseteq S\left(M_{\alpha}\right)=0$. Hence $\beta$ is a welldefined map, which is an $(R-)$ homomorphism. Also, it is clear that $\beta$ is an $R / S(R)$ homomorphism. Since $M_{\alpha}$ is $R / S(R)$-injective, there is an $m \in M_{\alpha}$, such that $\beta(x+S(R))=m(x+S(R))$, for all $(x+S(R)) \in(I+S(R)) / S(R)$. Thus, we have $\alpha(x)=m x$, for all $x \in I$. Hence $M_{\alpha}$ is $(R-)$ injective. Therefore, $M=\bigoplus_{\alpha \in \Lambda} M_{\alpha}$ is a direct sum of torsion injective modules. Now, since $R$ has ACC on $\mathscr{F}$-ideals, it follows from Proposition 1 that $M$ is injective.

Now we recall a definition. $R$ is called a left $T$-ring if every non-zero left $R$-module has non-zero socle. For the purpose of the next theorem, we need a definition which is weaker than that of left $T$-rings. Namely, rings for which every non-zero torsion cyclic module has non-zero socle. Such rings will be called weakly $T$-rings.

THEOREM 3. Let $R$ be a weakly T-ring, and $(G, F)$ be the Goldie torsion theory for $R$-Mod. Then the following are equivalent:

(1) $R$ is a left TQI-ring;

(2) $R$ has ACC on $\mathscr{F}$-ideals and each $\mathscr{F}$-ideal is the intersection of maximal left ideals ;

(3) the torsion class coincides with the class of semisimple modules.

Proof. (1) $\Rightarrow(2)$. This follows from Corollary 1 to Lemma 2 and Lemma 3, and does not depend on the assumption that $R$ is a weakly $T$-ring.

(2) $\Rightarrow(3)$. Let us first consider a torsion quasi-injective left $R$-module $M$. By Lemma $5, M=\bigoplus_{\alpha} M_{\alpha}$, where each $M_{\alpha}$ is a torsion indecomposable quasi-injective left $R$-module. Now consider an arbitrary but fixed $M_{\alpha}$. Let $x \in M_{\alpha}, x \neq 0$. Then $R x$ is a non-zero torsion cyclic submodule of $M_{\alpha}$. Since $R$ is a weakly $T$-ring, $R x$ has non-zero socle. So, let $S$ be a non-zero simple torsion submodule of $R x$. By Corollary 2 to Lemma 2, it follows that $S$ is injective. Hence $S$ is a direct summand of $M_{\alpha}$. But $M_{\alpha}$ is indecomposable. So, $M_{\alpha}=S$. Thus $M=\oplus M_{\alpha}$ is a direct sum of torsion injective simple modules. Hence $M$ is semisimple, and also injective by Proposition 1 . Since for the Goldie torsion class, every torsion module is a submodule of a torsion injective module, it follows that every torsion module is semisimple.

$(3) \Rightarrow(1)$. This is immediate.

We now give an example of a left TQI-ring which is not a left QI-ring.

ExAmple 1. Let $F$ be a field, and let $R=\left[\begin{array}{ll}F & 0 \\ F & F\end{array}\right]$. Also, let $A=\left[\begin{array}{ll}F & 0 \\ F & 0\end{array}\right]$ and $B=\left[\begin{array}{ll}0 & 0 \\ F & F\end{array}\right]$.

In the above example, $Z\left({ }_{R} R\right)=0$, and $B$ is the unique proper essential left ideal of $R$, which is also a maximal left ideal. Clearly, $R$ is an Artinian ring. Thus $R$ is a left $T$-ring 


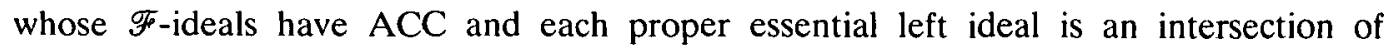
maximal ideals. Now consider the Goldie torsion theory over $R$. Since $Z\left({ }_{R} R\right)=0$, the associated filter $\mathscr{F}(G)$ consists of essential left ideals of $R$. Hence by Theorem 2 (statement (2)), $R$ is a left TQI-ring. On the other hand, since $J(R)=A \cap B \neq(0), R$ is not a $V$-ring. Hence there exists a simple left $R$-module which is not injective. Since simple modules are quasi-injective, it follows that $R$ is not a left QI-ring.

The purpose of the next theorem is to obtain a characterization of semilocal TQI-rings. By a semilocal ring we mean a ring which contains only a finite number of distinct maximal left ideals.

THEOREM 4. Let $R$ be a semilocal ring. Then the following are equivalent:

(1) $R$ is left TQI;

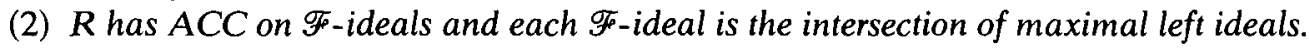

Proof. The implication (1) $\Rightarrow(2)$ follows Corollary 1 to Lemma 2 and Lemma 3, and it does not depend on the hypothesis that $R$ is semilocal. Hence we need only to prove (2) $\Rightarrow(1)$. Let $M$ be a torsion quasi-iniective left $R$-module. Then, by Lemma $5, M=$ $\bigoplus_{\alpha} M_{\alpha}$, where each $M_{\alpha}$ is a (torsion) indecomposable quasi-injective left $R$-module. Consider an arbitrary but fixed $M_{\alpha}$. Let $x \in M_{\alpha}, x \neq 0$. If $R x \cong R$ then $M_{\alpha}$ contains a copy of $R$. Since $M_{\alpha}$ is quasi-injective, an application of Baer's criterion implies that $M_{\alpha}$ is injective. Now suppose $R x \cong R / I$, for some left ideal $I$ of $R$. Then $I$ is an $\mathscr{F}$-ideal. Hence there exist maximal left ideals $M_{1}, \ldots, M_{n}$ of $R$ such that $I=M_{1} \cap \ldots \cap M_{n}$. We claim that $R / I$ is semisimple Artinian. Let us define a map $\phi: R / I \rightarrow R / M_{1} \oplus \ldots \oplus R / M_{n}$, by $\phi(\bar{r})=$ $\left(\bar{r}_{1}, \ldots, \bar{r}_{n}\right)$, where $\bar{r}=r+I$ and $\bar{r}_{k}=r+M_{k}, k=1, \ldots, n$. Clearly, $\phi$ is an $R-$ homomorphism. Also, $\phi$ is an injection because for each non-zero $\bar{r} \in R / I$, there is some $M_{i}$ such that $r \notin M_{i}$. Thus $R / I$ is a submodule of a semisimple Artinian module. Hence $R / I$ is semisimple Artinian. As shown in Corollary 2 to Lemma 2, if each $\mathscr{F}_{\text {-ideal }}$ is the intersection of maximal left ideals then each torsion simple left $R$-module is injective. Hence $R / I$ is injective. This implies that $R x$ is a direct summand of $M_{\alpha}$. But $M_{\alpha}$ is indecomposable. Hence $M_{\alpha}$ is injective. Consequently, $M=\bigoplus M_{\alpha}$ is injective, by Proposition 1 , as $R$ has ACC on $\mathscr{F}_{\text {-ideals. }}$

We shall now study commutative TQI-rings. Let us first recall that a ring $R$ is said to have $S P$ if every left $R$-module splits (i.e. if the torsion submodule of each left $R$-module $M$ is a direct summand of $M$ ). The next lemma is proved for not necessarily commutative TQI-rings.

LEMma 6. Let $R$ be a left TQI-ring. Then every $\mathscr{F}_{\text {-ideal }} R$ is idempotent.

Proof. Let $I$ be an $\mathscr{F}$-ideal of $R$. If $I=I^{2}$ then there is nothing to prove. So, suppose $I \neq I^{2}$. Since, for each $r \in I, I\left(r+I^{2}\right)=0$ in $I / I^{2}$, i.e. $I\left(I / I^{2}\right)=0$, it follows that $I / I^{2}$ is torsion. Now consider the exact sequence: $0 \rightarrow I / I^{2} \rightarrow R / I^{2} \rightarrow R / I \rightarrow 0$. Since $I / I^{2}$ and $R / I$ are torsion, $R / I^{2}$ is torsion. Hence $I^{2}$ is an $\mathscr{F}$-ideal. Hence by Corollary 1 to Lemma 1 , both $I$ and $I^{2}$ are intersections of maximal left ideals. Thus there is a maximal left ideal $M$ of $R$ 
such that $I^{2} \subseteq M$ but $I \nsubseteq M$. Since $M$ is a maximal ideal, $R=I+M$ and $1=x+m$, for some $x \in I$ and $m \in M$. Then $x=x^{2}+x m \in M$. Thus $1 \in M$, which is a contradiction.

LEMMA 7. Every commutative TQI-ring is a $\mathrm{V}$-ring (and hence a regular ring).

Proof. It is enough to show that $I=I^{2}$, for each ideal $I$ of $R$. If $I$ is an essential ideal

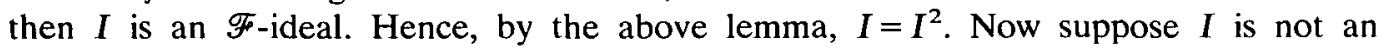
essential ideal, then there is an ideal $J$ of $R$ such that $I \cap J=(0)$ and $(I+J) \subseteq \subseteq^{\prime} R$. Then $(I+J)=(I+J)^{2}=I^{2}+I J+I J+J^{2}=I^{2}+J^{2}$. This equation implies that $I=I^{2}$.

The next lemma is due to Cateforis and Sandomierski [5, Theorem 2.1, p. 156].

LEMMA 8. For a commutative ring $R$, the following are equivalent:

(1) $R$ has $S P$;

(2) $Z(R)=0$ and for every essential ideal I of $R$, the ring $R / I$ is semisimple Artinian;

(3) every $R$-module $M$ with $Z(M)=M$ is $R$-injective. In particular, if $R$ has $S P$ then $R$ is hereditary.

THEOREM 5. Let $R$ be a commutative ring and $(G, F)$ be the Goldie torsion theory for $R$-Mod. Then the following are equivalent:

(1) $R$ is TQI;

(2) $R$ has $S P$.

Proof. Let us assume (1). Then, by Lemma $7, R$ is regular. Hence $Z(R)=0$. Now let $I$ be an essential ideal of $R$. Then $R / I$ is a regular ring. Since $R$ is a TQI-ring, $R$ has ACC on $\mathscr{F}$-ideals by Lemma 3. Further, since every essential ideal of $R$ is an $\mathscr{F}$-ideal, it follows that $R / I$ is Noetherian, as an $R$-module and hence as an $R / I$-module. Thus $R / I$ is a regular Noetherian ring. Hence $R / I$ is a semisimple Artinian ring. Therefore, $R$ has SP by Lemma 8 (statement (2)). Let us now assume that $R$ has SP. Then $R$ is hereditary by Lemma 8. Hence $Z(R)=0$. Thus the class of torsion modules, relative to the Goldie torsion theory for $R$-Mod, are precisely those modules $M$ for which $Z(M)=M$. But such modules are injective by Lemma 8 . Hence, in particular, torsion quasi-injective modules are injective. So, $R$ is a TQI-ring.

We now give an example of a commutative TQI-ring which is not a QI-ring.

ExAmple 2. [5, p. 161]. Let $K$ be a field and $A$ an infinite indexing set. Let $Q=\prod_{\alpha \in A} K^{(\alpha)}$, where $K^{(\alpha)}=K$ and $R=\sum_{\alpha \in A} \oplus K^{(\alpha)}+1 K \subseteq Q, 1 \in Q$. Then $R$ has only one essential ideal, namely, $I=\sum_{\alpha \in \mathrm{A}} \oplus K^{(\alpha)}$, and $I$ is maximal. Since $R$ is regular, $R$ has SP by Lemma 8 . Hence $R$ is a TQI-ring by Theorem 5 . On the other hand, since $R$ is not semisimple Artinian and since commutative QI-rings are necessarily semisimple Artinian, it follows that $R$ is not a QI-ring.

As noted earlier, commutative TQI-rings are hereditary. Thus the next proposition is an immediate consequence of the following result, which is also due to Cateforis and Sandomierski [5, Theorem 4.1, p. 164]. 
LEMMA 9. Let $\left\{R_{\alpha} \mid \alpha \in \Lambda\right.$ and $R_{\alpha} \neq 0$ for all $\left.\alpha\right\}$ be an infinite collection of hereditary rings $R_{\alpha}$ (with identity). Then $\prod_{\alpha} R_{\alpha}$ is not a hereditary ring.

Propostrion 2. An infinite direct product of commutative TQI-rings cannot be a TQI-ring.

We now characterize rings whose torsion quasi-injective left $R$-modules are $\Sigma$-quasiinjective. Recall that a quasi-injective module $M$ is $\Sigma$-quasi-injective if $M^{(\mathrm{A})}$ (the direct sum of card $A$ copies of $M$ ) is also quasi-injective, for any set $A$. First we state two needed results.

Lemma 10. [14, Prop. 4.2, p. 21]. Let $(T, F)$ be any stable torsion theory for $R$-Mod (i.e. $T$ is closed under injective hulls). Then every injective left $R$-module splits.

LemMA 11. [9, Cor. 2.2]. If $M$ is quasi-injective and $E(M)^{(A)}$ is injective then $M^{(A)}$ is quasi-injective for any set $A$.

THEOREM 6. Let $R$ be any ring and $(G, F)$ be the Goldie torsion theory for $R$-Mod. Then the following are equivalent:

(1) each torsion quasi-injective left $R$-module is $\Sigma$-quasi-injective;

(2) $R$ has ACC on $\mathscr{F}$-ideals.

Proof. (1) Suppose each torsion quasi-injective left $R$-module is $\Sigma$-quasi-injective. Let $I_{1} \subseteq I_{2} \subseteq \ldots \subseteq I_{k} \subseteq \ldots$ be an ascending chain of $\mathscr{F}$-ideals of $R$. Then we get the ascending chain

$$
(0)=I_{1} / I_{1} \subseteq I_{2} / I_{1} \subseteq \ldots \subseteq I_{k} / I_{1} \subseteq \ldots
$$

of submodules of the left $R$-module $\bar{R}=R / I_{1}$. Let $\bar{I}_{k}=I_{k} / I_{1}, k=1,2, \ldots$ Now consider the left $R$-modules $\bar{R} / \bar{I}_{k}$. Clearly, each $\bar{R} / \bar{I}_{k}\left(\cong R / I_{k}\right)$ is a torsion $R$-module. Let $Q_{i}=$ $E\left(\bar{R} / \bar{I}_{i}\right)$. Then each $Q_{i}$ is a torsion left $R$-module. Let $Q=\bigoplus_{i} Q_{j}$ and $M=\prod_{j} Q_{j}$. Since $M$ is injective, Lemma 10 implies that $M$ splits. Suppose $M=T \oplus K$, where $T$ and $K$ are the torsion and torsion free parts of $M$, respectively. Since each $Q_{j} \subseteq T$ and each $Q_{j}$ is injective, we may write $T=T_{i}=Q_{j} \oplus P_{i}$, for some $P_{j} \subseteq T$. Hence $\underset{i}{\bigoplus_{j}} T_{j} \bigoplus_{i} Q_{i} \oplus \underset{i}{Q_{j}} P_{j}$. Thus $Q=\bigoplus_{j} Q_{j}$ is a direct summand of $\underset{j}{\bigoplus_{j}} T_{j}$. But $\underset{j}{\bigoplus_{j}} T_{j}$ is quasi-injective by the hypothesis. Hence $Q$ is quasi-injective. Now let $\bar{I}=\bigcup_{k=1}^{\infty} \bar{I}_{k}$. Then the natural $(R-)$ homomorphism $f_{k}: \bar{I} \rightarrow \bar{R} / \bar{I}_{k}$ maps $\bar{I}$ into $Q_{k}$. Note that if $a \in \bar{I}$ then $a \in \bar{I}_{t}$ for some t. Thus $f_{k}(a)=0$, for all $k \geq t$. Let us now define a map $f: \bar{I} \rightarrow Q$ by $f(a)=$ $\left(f_{1}(a), \ldots, f_{i}(a), \ldots\right), a \in \bar{I}$. This definition is meaningful since only a finite number of terms on the right-hand side are non-zero. Clearly, $f$ is an $R$-homomorphism. Now $\bar{I} \subseteq \bar{R} \subseteq Q_{1} \subseteq Q$, and $Q$ is quasi-injective. Hence there is a map $\lambda \in \operatorname{Hom}_{R}(Q, Q)$ which induces $f$. Thus $f(\bar{I}) \subseteq \lambda(\bar{R}) \subseteq Q$. Suppose $\lambda\left(1+I_{1}\right)=m$, where 1 is the identity of $R$. Now 
$m \in \sum_{i=1}^{t} Q_{i}$, for some $t$. Hence $f(\bar{I}) \subseteq R m \subseteq \sum_{i=1}^{t} Q_{i}$. This implies that $\bar{I}_{t+1}=\bar{I}_{t+2}=\ldots=\bar{I}$. Hence $I_{t+1}=I_{t+2}=\ldots=I$. This proves that $R$ has ACC on $\mathscr{F}$-ideals.

(2) Let us now suppose that $R$ has ACC on $\mathscr{F}$-ideals, and let $M$ be any torsion quasi-injective left $R$-module. Then $E(M)$ is torsion, as we are working in the Goldie torsion theory. Moreover, since $R$ has ACC on $\mathscr{F}$-ideals, $E(M)^{(\mathrm{A})}$ is injective, for any set $A$, by Proposition 1 . Hence, by Lemma $11, M^{(A)}$ is quasi-injective, for any set $A$.

ACKNowledgment. The first named author takes this opportunity to thank the Mathematics Department of the University of Kentucky for its hospitality during his visit.

\section{REFERENCES}

1. A. K. Boyle, Hereditary QI-rings, Trans. Amer. Math. Soc. 192 (1974), 115-120.

2. A. K. Boyle and K. R. Goodearl, Rings over which certain modules are injective, Pacific $J$. Math. 58 (1975), 43-53.

3. K. A. Byrd, When are quasi-injectives injective?, Canad. Math. Bull. 15 (1972), 599-600.

4. K. A. Byrd, Rings whose quasi-injective modules are injective, Proc. Amer. Math. Soc. 33 (1972), 235-240.

5. V. C. Cateforis and F. L. Sandomierski, The singular submodule splits off, J. Algebra 10 (1968), 149-165.

6. J. Cozzens and C. Faith, Simple noetherian rings (Cambridge Univ. Press, 1975).

7. C. Faith, Modules finite over endomorphism rings, Lectures on rings and modules, Lecture Notes in Mathematics 246 (Springer, 1972), 146-189.

8. C. Faith, On hereditary rings and Boyle's conjecture, Arch. Math. 27 (1976), 113-119.

9. K. R. Fuller, On direct representations of quasi-injectives and quasi-projectives, Arch. Math. 20 (1969), 495-502.

10. J. Golan and Z. Papp, Cocritically nice rings and Boyle's conjecture, Comm. Algebra 8 (1980), 1775-1798.

11. J. Golan and M. Teply, Finiteness conditions on filters of left ideals, J. Pure and Applied Algebra 3 (1973), 251-260.

12. J. Lambek, Torsion theories, additive semantics and rings of quotients, Lecture Notes in Mathematics 177 (Springer, 1971).

13. G. O. Michler and O. E. Villamayor, On rings whose simple modules are injective, $J$. Algebra 25 (1973), 185-201. 1971).

14. B. Stenström, Rings and modules of quotients, Lecture Notes in Mathematics 237 (Springer,

Department of Mathematics

UNIVERSTTY OF KENTUCKY

LEXINGTON, KENTUCKY 40506

U.S.A. 